\title{
“Green" Supply Chains: Using Information Integration for Sustainable Development
}

\author{
Alexander Schatten \\ Vienna University of Technology \\ Institute for Software Technology and Interactive Systems \\ Favoritenstr. 9-11/188, 1040 Vienna, Austria \\ schatten@ifs.tuwien.ac.at
}

\begin{abstract}
The current development of global economies and societies is regarded as being highly unsustainable. This paper analyses the current situation. Instead of simplistic metrics that take only snapshots of the life-cycle of a product into consideration, an approach is introduced that covers the whole supply chain. The core idea is that end-to-end transparency, specific data models, a process architecture, modern data management and analysis techniques are the key to make informed decisions, optimisations and to execute international policies. The particular role of ICT in such a future economy is discussed.
\end{abstract}

\section{Introduction}

Information and Communication Technology (ICT) is not (as sometimes believed) per se a "green" technology. To move bits back and forth, a lot of infrastructure is actually required (e.g. servers, networks, devices). As a matter of fact, growth rates in ICT have been enormous in the last years and so have the negative impacts like resource and energy consumption or emission of greenhouse gases. Having said that, ICT provides on the other hand a series of opportunities as enabling technology to support other fields in reaching reductions in greenhouse gases that have the potential to overcompensate the emissions of ICT itself by far [13].

Sustainable Development will only be possible if we have a fine-grained overview on the whole process of production, starting with the resources and covering production lines and transport, usage and final recycling and disposal. Energy, resource and emission control and optimisation as well as accounting ("who" is responsible for "what amount") are only possible in case of a transparent process.

This paper outlines the potential of information integra- tion, modern data management and analysis techniques in providing transparent "green" supply chains that support sustainable development.

\section{Motivation}

The current status of our societies and economy is apparently highly unsustainable. This is no new finding, but it became more obvious with the rapidly growing economies of developing countries in Asia. Global economic growth amplified the effects on our unsustainable activities in many ways. The examples given in this section will illustrate that grown (global) supply chains are highly intransparent and result in a negative impact on local economies, efficiency and environment:

Direct Impact of ICT Despite all activities in the last years, greenhouse gas emission is high and still rising significantly every year. The emissions of just the ICT sector were approx. 0,53 billion tonnes (Gt) carbon dioxide equivalent $\left(\mathrm{CO}_{2} \mathrm{e}\right)$ in 2002. Assuming "business as usual", production is estimated to rise to approximately $1,4 \mathrm{Gt} \mathrm{CO}_{2} \mathrm{e}$ in 2020 [13].

Illegal Logging and Degradation of Biodiversity Illegal Logging is happening all over the world, mostly in developing countries. Loss of (primary) rain-forests is a major problem for biodiversity [7], water supply and the world's climate. Additionally, the financial loss for local communities in tax-losses can be substantial. Just the annual financial loss of Indonesia's government is estimated to be $\$ 3$ billion [2].

Inefficiencies in production automation The current status in production automation is not using the latest bestpractices and standards used, e.g. in business informatics. 


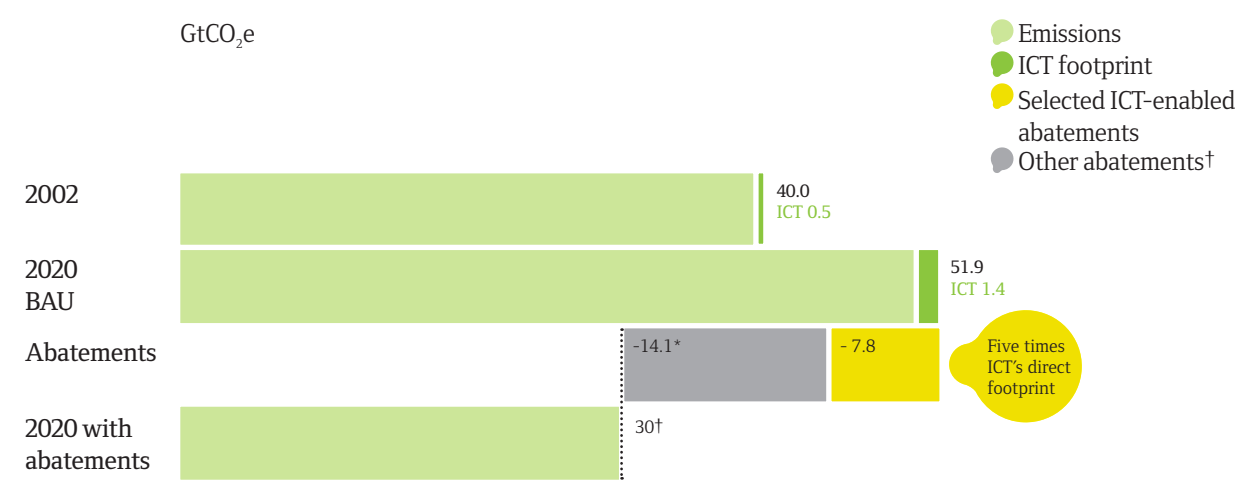

Figure 1. Estimation of greenhouse gas emissions in 2020, the ICT footprint and the assumed ICT enabled abatements. Taken from [13].

The result is that plants, production lines and factories are not as efficient, flexible and easy to monitor and control as desired. Optimisation is not easy on a factory level, not to mention the whole supply chain. Current research is focusing on raising control systems to a level that allows higher flexibility and efficiency due to better information integration between systems and better software engineering approaches (e.g., Heck et al. [6]).

Logistics Global transportation is increasing dramatically every year. In 2004 the growth rate of global trading of goods was 9\% [1]. This is not only an environmental problem, but also an economic one. Rising energy prices will have a significant impact on transportation costs on all carriers ("The cost of shipping a standard 40-foot container from Shanghai to America's east coast, for example, has jumped from \$3,000 in 2000 to about \$8,000 today." [3]). Optimisation and support for searching alternative scenarios are an important target.

Electronic Waste Disposal On the other end of the product life-cycle is waste. Ideally, products at the end of their life-cycle can be recycled. More likely is down-cycling, regular disposal or questionable channels in which waste is "disappearing". Electronic waste recently has a particular bad reputation as large amounts are dumped in developing countries where locals poison themselves and the environment by non-professional dismantling and "recycling" attempts [5].

\section{Amplifying factors}

In addition to the problems mentioned above, there are amplifying factors and boundary conditions that need to be taken into consideration as well. A fundamental issue is the lack of transparency and accountability: many criminal activities like illegal logging or smuggling are "under the radar" of the authorities or are ignored due to corruption. Some are detected (like illegal logging), but too late, so that the damage is done. If such activities are eventually revealed, the responsible parties often cannot be identified any more; and even if illegal activities are revealed and the local person responsible is identified, this person or group is actually only a tiny part of the chain; the person responsible for shipping, the person who made the profit, the customers owning the devices, etc. are are all part of the problem.

Information distribution and aggregation is often not done, or done in difficult manual studies (e.g., sites of illegal waste disposal, smuggling of endangered species or forest degradation). But in most cases, aggregated (near) real time information that is also sufficient to provide accountable information would be required to act quickly and detect the chains of responsibility. These issues are nearly always systemic problems and not failure of one individual.

In close connection with these issues is obviously corruption, that is prevalent in many developing countries like Indonesia [12]. There is an inner resistance against change as many officials profit from illegal activities. As a consequence it is often very difficult to enforce international regulations or policies. Even if procurement policies for some companies exclude e.g. the use of wood from nonsustainable forestry, can the "certification" or promises of the supplier be trusted?

Finally, we tend to over-simplify problems and reduce complex systemic issues to primitive metrics. The recent discussion about the environmental impact of cars, for example, is often limited to the fuel consumption or the emission of $\mathrm{CO}_{2} \mathrm{e}$. The impact of a car certainly is depending on many other factors like the energy and resource consumption during production or the support infrastructure (streets, gas-stations, etc.). To calculate more significant metrics, 
several suggestions have been made, among them the calculation of material input per service unit (MIPS). The calculation is, however, not simple; particularly the gathering of the underlying data: "The gathering of data (and the verifying thereof) is the most important and frequently the most time-consuming step of the MIPS analysis." [11]. The idea of calculating such metrics near realtime is of course out of question as yet.

The usage of overly simplified metrics can lead to a quite biased picture of the actual situation and is not helpful; particularly because optimisation efforts tend to be expensively implemented on sub-optimal places in the supply chain, whereas "lower hanging fruits" are easily overseen.

\section{Opportunities and the Role of ICT}

The Smart 2020 study [13] reports current $\mathrm{CO}_{2} \mathrm{e}$ greenhouse gas emissions and predictions for different branches for 2020. Needless to say, predictions under "business as usual" assumptions show strong growth. On the other hand, the huge potential to higher efficiency (see also Lovins et.al. [9]) also driven by clever usage of ICT is outlined by this report (see fig. 1).

Furthermore, recognising the global problems, the European Union and other governments introduce new regulations and production guidelines and policies. The European Commission currently tries to negotiate deals with certain governments about sustainable forestry [2]. Even investment banks like Goldman Sachs introduce policies of that sort [4]. However, policies are one thing; to enforce these regulations, a tight control scheme and certification process are required as will be explained in the next section.

Yet a lot of monitoring capabilities are already available or will be in place soon. Just in the European Union a series of observation programs are started like Kopernikus land information services, INSPIRE and the global monitoring for environment and security GMES.Such services have the potential to provide essential information that are needed for a transparent supply chain, like observation of forests and fishery. Initial projects in Africa are already in place.

Also efforts to optimise production automation are currently a hot $R \& D$ topic. Yet the integration of data from other resources into production systems and from production systems in the supply chain are still a significant challenge.

And finally, eGovernment efforts are spreading all over the world, also in developing countries like India or Indonesia. The impact of connected eGovernment applications in the context of "green" supply chains can be substantial for example in increasing public awareness for local problems and fighting illegal activities and corruption. Tanmoy Chakrabarty, vice president of Government Industry Solutions at Tata Consultancy Services (TCS) in India

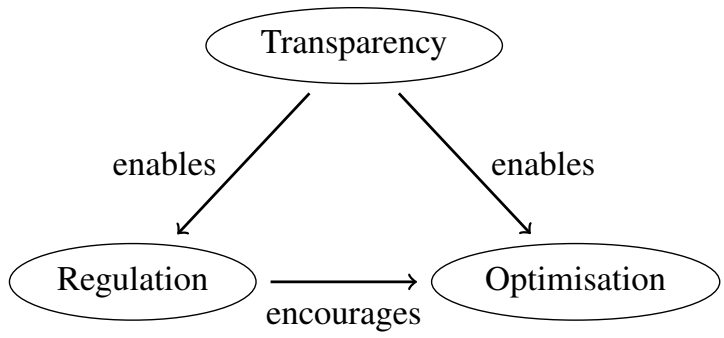

Figure 2. Transparency is the foundation for optimisation and regulation/certification efforts

says in an interview: "Corruption is the biggest enemy of e-government" [10]. When seen the other way round, implementation of e-Government, particularly connected with other international information systems is a very potent medication against corruption and illegal activities.

\section{Research Issues}

As mentioned above, a number of pieces that can lead to traceable supply chains are already available. But clearly a lot is still missing. On the domain side (like in production domain), activities need to be done on two levels: (1) infrastructure has to be modified so that information exchange on standardised protocols is possible, and (2) systems should be enabled to react fast to changes in the environment (indicated by external information). The first point is more important and also the more realistic one in the short run. Even "conservative" domains like production automation are moving towards e.g. internet protocols for communication between systems.

To go ahead an important next step, it is suggested to define integration protocols, data models and a reference architecture that have to fulfil several conditions, at least the following:

- allows to model items (e.g. a piece of wood, a transistor), collections/compositions of items ("a notebook"), transformation of items ("wood is cut or burned"), inheritance etc.

- supports a distributed infrastructure

- defines services for different stakeholders

- deals with certified and uncertified partners, certification authorities

- allows distributed queries ("show history of product X") 
- allows assertions and checks on transition points (e.g. to enforce regulations)

- defines a (distributed) process model

- supports connections (links) to various other data resources like the mentioned geographical information systems

- is integrated into available tracking technologies like RFID

- is openly specified and provides open source implementations for easy implementation

- allows gradual adoption

On this basis concrete systems can be implemented that support (among others) the verification of regulations, tracking down of counterfeits, supporting optimisation on global criteria like MIPS. Also powerful data management and analysis techniques can be applied for reporting and near real-time actions (sense and respond).

Already in the absence of such an infrastructure, modern analysis techniques can help providing indirect evidence using yet available information. This could be already demonstrated in 1995 by Jack L. King. He provided indirect evidence to undeclared nuclear activities by analysing various data sources and creating indirect evidence: "Treaty verification requires accurate and complete information describing the relevant activities of the signatories. This implies not only that all activities are declared and described accurately by the parties, but also that no activities exist which have not been declared. Assurance on the absence of undeclared activities can not be obtained through direct verification, and therefore an approach which includes qualitative analysis of information about their possible existence is proposed." [8]

One has to keep in mind that in 1995 not even the Internet was available, providing the scale of public information accessible today; not to mention an established connected information systems for transparent supply chains as suggested here.

\section{Conclusion}

The previous sections outlined the importance of transparent supply chains to aim for a sustainable economy. Integration of a large number of data sources and application of modern analysis techniques (e.g. data warehouses) is one essential piece of the solution. On the one hand integrated systems should allow end-to-end monitoring of supply-chains on a high level (e.g. governmental, trade organisations or UN) but on the other hand, these information systems should enable also grass-root activities in understanding what is happening in a particular place in the world ("Village Driven Accountability" [12]), e.g. fighting the abuse of natural resources in Africa. (Fig. 2 shows the core idea: increased transparency enables optimisation efforts and international regulations). The goal is clearly global over local optimisation.

But optimisation is not the only issue here; maybe (in the long run) even more important is the fact that a proper accounting and cost transparency, i.e. which part of the supply chain is responsible for what amount of a broad range of resource consumption, production of greenhouse gases, production of pollutants of other sorts and treatment of waste.

Reliability and traceability of supply chains lay the foundation for "total end-to-end cost" calculations like MIPS and taxation, $\mathrm{CO}_{2} \mathrm{e}$ balances and so on, helping in tracking down responsibilities and in finding better alternatives to practices we are using now.

\section{References}

[1] Boom and bust at sea. The Economist, August 2005.

[2] Down in the woods. The Economist, May 2006.

[3] High seas, high prices. The Economist, August 2008.

[4] Goldman sachs environmental policy framework. http: //www2.goldmansachs.com/citizenship/ environment/policy-framework.pdf.

[5] Poisoning the poor, electronic waste in ghana. http:// www.greenpeace.org/international/press/ reports/poisoning-the-poor-electonic, August 2008. Greenpeace Report.

[6] B. Heck, L. Wills, and G. Vachtsevanos. Software technology for implementing reusable, distributed control systems. Control Systems Magazine, IEEE, 23(1):21-35, Feb 2003.

[7] J. Barlow et.al. Quantifying the biodiversity value of tropical primary, secondary, and plantation forests. Proceedings of the National Academy of Sciences of the United States of America, 104(47), Nov 2007.

[8] J. L. King. Propagation and Visualization of Evidence An Interactive Qualitative Analysis System for the Detection of Undeclared Nuclear Activities. PhD thesis, University of Vienna, 1995.

[9] A. B. Lovins, E. K. Datta, O.-E. Bustnes, J. G. Koomey, and N. J. Glasgow. Winning the Oil Endgame. Rocky Mountain Institute, Snowmass, Colorado, 2005.

[10] S. Prasad. Corruption slowing india's e-govt growth. http: //www. zdnetasia.com/news/business / 0, 39044229,62044787,00.htm, 2008.

[11] M. Ritthoff, H. Rohn, C. Liedtke, and T. Mertens. Calculating mips, resource productivity of products and services. Technical report, Wuppertal Institute for Climate, Environment and Energy, 2002.

[12] J. ud-din Kassum, A. Steer, H. Kharas, and J. Hellman. Indonesia: Combating corruption in indonesia, enhancing accountability for development. Technical report, World Bank, November 2003.

[13] M. Webb. Smart 2020: Enabling the low carbon economy in the information age. http://www . theclimategroup.org, 2008. 